Results Questionnaire response rate: 69/487=14\%. Sudden, unexpected deaths: $33 / 69=48 \%$.

Sudden deaths communicated in face-to-face meetings: 28/ $33=85 \%$. By telephone: $5 / 33=15 \%$.

Location of disclosure: at bedside $22 / 28=79 \%$, private room $6 / 28=21 \%$. Communication by: consultant $18 / 33=55 \%$; nurses $8 / 33=24 \%$; trainee doctor $1 / 33=3 \%$; others $6 /$ $33=18 \%$.

Communication empathetic and caring 33/33=100\%. Time of disclosure: immediately on arrival $17 / 33=52 \%$; within 30 minutes $7 / 33=21 \%$; within 1 hour $2 / 33=6 \%$; within $1-2$ hours $2 / 33=6 \%$; $>2$ hours after arrival $2 / 33=6 \%$ and present at 'death $3 / 33=9 \%$. Full explanation of the cause of death offered $32 / 33=97 \%$. Sufficient time for questions 32/ $33=97 \%$. Allowed to view deceased: $32 / 33=97 \%$.

Explanation for referral to Coroner: $14 / 21=67 \%$. Religious and cultural observances facilitated: $16 / 33=48 \%$. Organ and tissue donation discussed $7 / 33=21 \%$.

Conclusion In general, the process of disclosing unexpected hospital deaths to relatives is done well. Improvements could be made by expediting family meetings, prioritising privacy, discussing tissue donation and facilitating religious and cultural observances. A national guideline would be useful.

\section{UNDERSTANDING CURRENT PRACTICE IN PRESCRIBING AND USING ANTICIPATORY MEDICINES AT THE END OF LIFE}

Ben Bowers, Kristian Pollock, Stephen Barclay. University of Cambridge, University of Nottingham

\subsection{6/spcare-2020-PCC.70}

Background Anticipatory medications (AMs) are injectable drugs prescribed to a named patient, ahead of likely clinical need, for administration by visiting General Practitioners (GPs) and Community Nurses if symptoms arise in the final days of life. While the prescribing of AMs is recommended best practice in the UK, little is known about how they are used in primary care.

Aim To investigate primary care practice in issuing and using AMs for patients approaching the end of life.

Methods Retrospective review of the GP and Community Nursing records of 240 sequential patient deaths in eight GP practices in two UK counties (30 deaths per practice). Analysis used descriptive statistics and constant comparison between patient cases.

Results 132/240 (55\%) of deceased patients were issued AMs, between 695 and 0 days before death (median 17 days across all eight practices, range of median 38 days to 6 days in individual GP practices). AMs were often issued alongside completion of a DNACPR form: documented discussions centred on patients expressing preferences to stay at home and avoid hospital admission.

$79 / 132(60 \%)$ of the patients issued AMs went on to have them administered, almost exclusively by Community Nurses. Drugs were first used between 586 and 0 days before death (median 3 days). AMs were typically used when patients were identified as dying and unable to swallow oral medications. However, for 10/79 (13\%) of patient where AMs were used, they were started more than 2 months before death for reversible conditions and/or not at the end of life.
Conclusions The issuing and use of AMs were commonplace in all the GP practices, with considerable variation in timing between GP practices and community nursing teams. The findings highlight the challenges in diagnosing dying and the risks involved in prescribing AMs far in advance of likely need.

\section{SYSTEMATIC REVIEW LOOKING AT HOW EFFECTIVE CLINICIANS ARE AT PROGNOSTICATION AT THE END OF LIFE - TEMPORAL AND PROBABILISTIC}

C Campion, J Grant, N Shah, J Riley, J Droney. Royal Marsden NHS Trust - Coordinate my Care

\subsection{6/spcare-2020-PCC.71}

Background Clinician's estimation of patient survival can influence decisions regarding treatment, enable patients to make plans, improve quality of life and increase meeting preferred place of care and death. The two survival predictors frequently used are temporal and probabilistic. Temporal-the patient is predicted to live a certain amount of time, probabilistic-the chance of a person surviving to a certain time.

Aims Describe the published evidence, relating to the effectiveness and accuracy of clinicians at predicting clinical survival in patients with cancer and non-cancer.

Methodology The databases Embase, Cinahl, Medline and Emcare were searched using the terms 'prognosis', 'prognostication', 'surprise question' and 'advanced care planning'. Duplicates were removed. 127 papers were identified, 40 papers were selected as relevant to clinical effectiveness of prognostication at end of life. Literature was reviewed using the Preferred Reporting Items for Systematic Reviews and Meta-Analyses principles.

Results Clinicians are overly optimistic in their estimated survival predictions (studies found by at least 50\%). Recent systematic reviews of clinician's survival predictions suggest that they were correct in only $25 \%$ of cancer patients by within a week. This accuracy of predictions increased to $70 \%$ when patients were in the last days to weeks of life. Predictions utilizing probabilistic measures over the temporal approach have been found to be significantly more accurate. A large meta-analysis looking at the accuracy of the 'surprise question' and outcomes in 22 studies showed that $75 \%$ of clinicians accurately predicted if a patient would die within 12 months.

Conclusion Clinician's prognostication predictions are complex and evidence shows that clinicians are often over optimistic in their estimated survival predictions. Clinicians are significantly more accurate in prognosticating survival with use of probabilistic measures than temporal approach.

\section{REHABILITATIVE PALLIATIVE CARE AS A HEALTH PROMOTING APPROACH: A PARTICIPATORY ACTION RESEARCH STUDY}

Karen L Clarke. St Michael's Hospice

\subsection{6/spcare-2020-PCC.72}

Background Health promoting palliative care (HPPC) considers dying and death as a social phenomenon where communities play an integral role. The dominant interpretation of HPPC in the UK has been through community engagement activities 